\title{
Orientation Mapping by Electron Channeling (OMEC) of Polycrystals Using a Stage-Rocked Big-Data Approach
}

\author{
Karl A. Hujsak ${ }^{1}$, Benjamin D. Myers ${ }^{1,2}$ and Vinayak P. Dravid ${ }^{1,2}$ \\ 1. Department of Materials Science and Engineering, Northwestern University, Evanston, IL. \\ 2. NUANCE Center, Northwestern University, Evanston, IL.
}

Obtaining an accurate and precise measure of orientation in a polycrystalline material is a crucial characterization step in making structure/property connections in functional materials. In the past, understanding polycrystalline texture was frequently explored using selected area electron channeling patterns (SACP). By tilting the electron beam in fine increments on a polished specimen, observed changes in BSE contrast can be described by a superposition of Bloch waves which are highly dependent on the orientation of the crystal lattice. Recording this intensity directly onto a sphere, a map of orientation space can be made. Tilting the electron beam to large angles while maintaining a fixed position was nontrivial and suffered from spherical aberration, limiting both the spatial resolution and angular field of view (FOV). Electron Backscatter Diffraction (EBSD) is relatively immune to these challenges, and with computational assistance has become a turnkey technique to produce high (although anisotropic) spatial resolution orientation maps. However, angular resolution has remained limited by the pixel density of the detector and the inherent geometric distortions from recording electrons from a tilted specimen onto an oblique detector. In this work, we will show how an orientation map complementary to EBSD can be generated from a stage-rocked sample in a conventional SEM, where full rastered BSE images are recorded at each tilt/rotation.

It was first noted over 50 years ago that electron channeling patterns (ECPs) could also be generated from stage-rocking with respect to a fixed electron beam [1]. This alternative to beam rocking was not put to practice given the experimental difficulties in routine eucentric rotation of a specimen with sub-micron precision. We propose that by recording a full BSE image at each tilt/rotation, computational methods can simultaneously be used to correct for stage errors while generating a full orientation map (many ECPs in parallel). In this way, the spatial resolution of the map is controlled by beam raster scan, and the angular resolution/FOV of each ECP is controlled by the programmed stage motion.

We first use automated image collection to generate a stack of images through a partial sphere of tilts/rotations with many unavoidable stage-induced positioning errors. These are corrected off-line utilizing a computational model which can account for translations, rotations, and perspective distortion by identifying corresponding points with a scale invariant feature transform (SIFT) [2]. The entire stack of images is then warped on to a single coordinate frame, and each pixel stack is mapped on to its own 3D sphere to produce an ECP for every pixel. Typically, tens-of-thousands of images are collected autonomously, which in turn can construct hundreds-of-thousands of individual ECPs.

We will show that ECPs generated by OMEC offer superior angular field of view compared to SACPs and sufficient angular resolution to enable direct indexing and/or potentially explore local strain/deformation. By directly measuring orientation information in three-dimensions, we avoid the geometric distortions from the gnomonic projection in EBSD, while requiring no dedicated detector. In addition, each BSE image contained in the stack has all the information in an electron channeling contrast image (ECCI). In OMEC, any number of ECCIs can be pulled out of the full series at an arbitrary number 
of diffraction conditions known exactly from the full ECP. We will also show that OMEC is amenable to acceleration using multi-objective dynamic sampling methods, in which the collection of orientationcritical BSE images is prioritized [3]. Thoughts for large area mapping with high angular precision and the prospects of data mining large sets of ECCIs at unique diffraction conditions will be discussed [4].

References:

[1] D. G. Coates, Philosophical Magazine 16 (1967), p. 1179.

[2] D. G. Lowe, The proceedings of the seventh IEEE international conference on Computer Vision (1999) p. 1150.

[3] G.M. Godaliyadda et al, Electronic Imaging 19 (2016), p. 1.

[4] This material is based on research sponsored by the Air Force Research laboratory under agreement No. FA8650-15-2-5518 and Air Force Office of Scientific Research under Award No. FA9550- 12-10280. This work made use of the EPIC facility of Northwestern University's NUANCE Center, which has received support from the Soft and Hybrid Nanotechnology Experimental (SHyNE) Resource (NSF ECCS-1542205); the MRSEC program (NSF DMR-1720139) at the Materials Research Center; the International Institute for Nanotechnology (IIN); the Keck Foundation; and the State of Illinois.
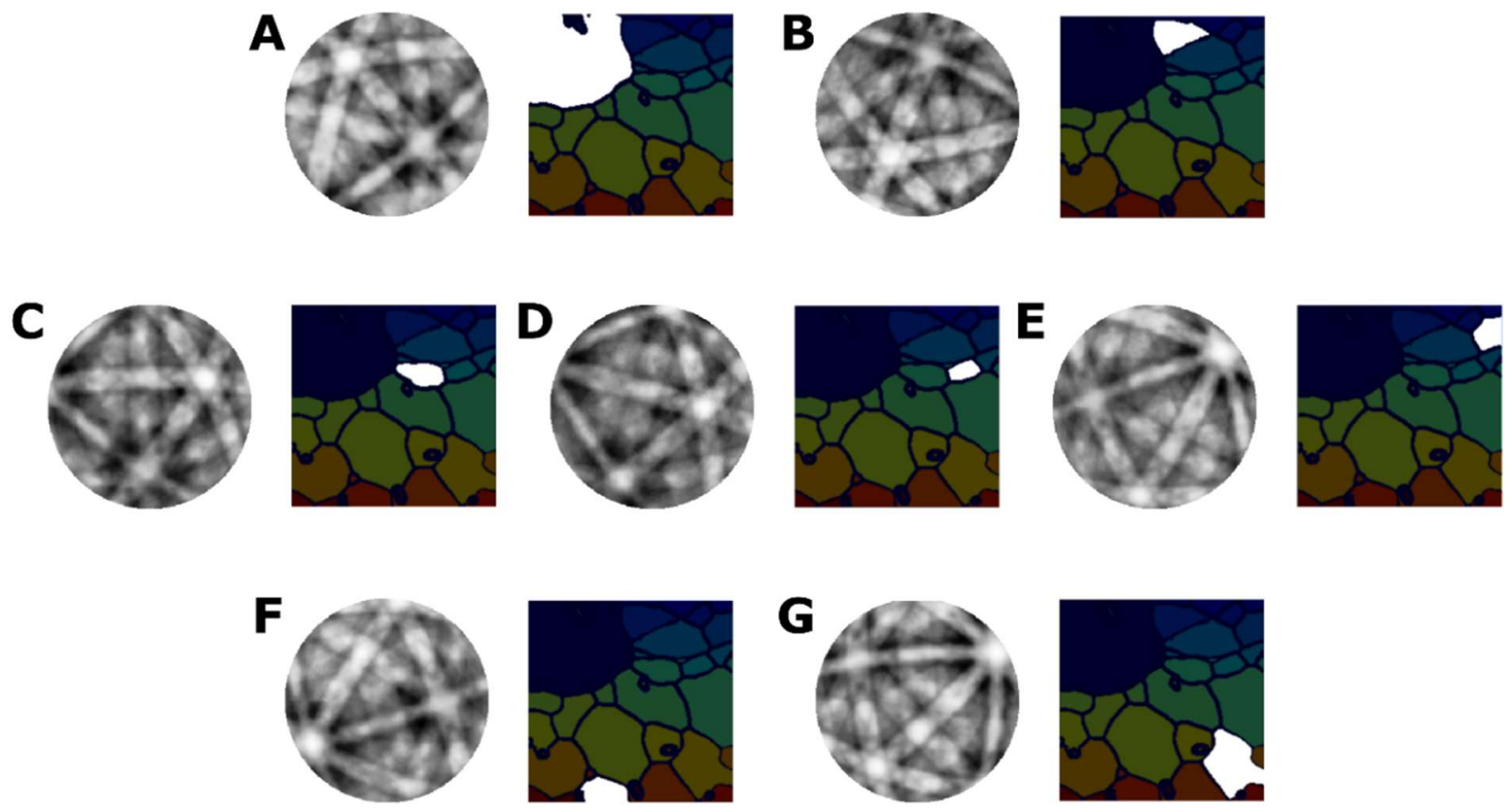

Figure 1. Example of experimental OMEC acquisition on a PbSe-GeSe polycrystalline thermoelectric material. For each sub-panel we have highlighted a grain out of the experimental orientation map generated from an OMEC experiment and the integrated stage-rocked ECP for that grain. The image stack was composed of 12,600 BSE images at 512,442 pixels. 